\title{
On the design, development and experimentation of the ASTRO assistive robot integrated in smart environments
}

\author{
Filippo Cavallo, Michela Aquilano, Manuele Bonaccorsi, Raffaele Limosani, Alessandro Manzi, \\ Maria Chiara Carrozza, Member, IEEE, and Paolo Dario, Member, IEEE
}

\begin{abstract}
This paper presents the full experience of designing, developing and testing ASTROMOBILE, a system composed of an enhanced robotic platform integrated in an Ambient Intelligent (AmI) infrastructure that was conceived to provide favourable independent living, improved quality of life and efficiency of care for senior citizens. The design and implementation of ASTRO robot was sustained by a multidisciplinary team in which technology developers, designers and end-user representatives collaborated using a user-centred design approach. The key point of this work is to demonstrate the general feasibility and scientific/technical effectiveness of a mobile robotic platform integrated in a smart environment and conceived to provide useful services to humans and in particular to elderly people in domestic environments. The main aspects faced in this paper are related to the design of the ASTRO's appearance and functionalities by means of a substantial analysis of users' requirements, the improvement of the ASTRO's behaviour by means of a smart sensor network able to share information with the robot (Ubiquitous Robotics) and the development of advanced human robot interfaces based on natural language.
\end{abstract}

\section{INTRODUCTION}

The Strategic Research Agenda in robotics predicted that, in future, robot co-workers will assist and cooperate closer with humans under many different circumstances. As consequence and necessity, future technologies will take in account improvements of interaction between humans and robots, independently of place (at work, in public, at home, on the moving), and both in tele-operated or individual task mode. The future vision of robotic co-workers also anticipated the idea of a companion robot, conceived as a robot assistant in domestic environments [1]. Nowadays this vision is tremendous true as consequence of the recent demographical trend. Indeed the European population projections have recently underlined that the number of senior citizens living in Europe will quickly increase in the coming years [2] and the Society will be necessarily forced to sustain them. Nowadays the Society sustains elderly people to age well by means of medical cures, socio-medical services, and other social activities but this request of support and assistance is so high that it is becoming difficult to manage and delivery because of the huge public welfare funds necessary for that and the current financial crisis [3]. Against these societal phenomena, Society is induced to

*Research supported by the ECHORD project, funded by the EU-FP7ICT-2007.2.2 (Agreement N.231143).

F. Cavallo, M. Aquilano, M. Bonaccorsi, R. Limosani, A. Manzi, M. C. Carrozza, and P. Dario are with the BioRobotics Institute of Scuola Superiore Sant'Anna, Pisa, Italy (corresponding author to provide phone: +39050883474; fax: +39050883101; e-mail: f.cavallo@sssup.it). think and revalue the figure and role of senior citizens and their relative socio-medical services. Beyond their demographical diffusion, seniors over 65 , the so called third age, are persons enough autonomous, living alone or with their partners and having some slight health problems. Preserving independence and favouring ageing well could induce important social and economical benefits, such as delaying and reducing institutionalization, increasing the labour force, favouring their participation to communities life, etc., and for this reason it is fundamental that societies take care of senior citizens from the beginning of their ageing.

Robotics and ICT technologies represents nowadays a profitable solution for more efficient management and delivery of health and social care, as well as improvement of independent living, quality of life and efficient cares of elderly people [4]. The idea of using robotic systems for assisting and monitoring people has been strongly supported by the European Commission through different research programs (FP7-ICT-Ch2/Ch5, AAL-JP, CIP-PSP, etc.) and shared by many research groups. Most of them have investigated the figure of robot for human assistance and studied all aspects related to the service provision, communication and safety interaction. Some examples of these projects are MOBISERV [5], a project conceived to enhance the independent living of elderly people and provide health care assistance and support in their daily social activities, KSERA [6], a project oriented to develop a domestic robot able to follow patients through the house, SRS [7], a project focused on the development of a semiautonomous robotic platform, FLORENCE [8], a project conceived to improve the well-being of elderly people as well as improve the efficiency in care through Ambient Assisted Living (AAL) services, COMPANIONABLE [9], a project focused on the integration of companion robots into smart home environments for care support to older persons (with Mild Cognitive Impairment) living alone at home and to their care-givers, Care-O-Bot [10] and the Robot Maid [11], two mobile robots to assist people in their home.

Differently from other works, the aims of this paper is to describe the full experience of designing, developing and testing the ASTROMOBILE System for favourable independent living, improved quality of life and efficiency of care and to demonstrate its the general feasibility, S/T effectiveness and acceptability by end-users. The ASTROMOBILE System was composed of the ASTRO Robot and a Wireless Sensor Network with environmental and wearable sensors, which was able to actively work in real conditions and cooperate with elderly. The objective of 
this work was to significantly enhance the quality and the acceptability of current robotic services to a new level of service and performance by:

- designing the ASTRO's appearance and functionalities by means of a substantial analysis of users' requirements and attitude to robotic technology to improve acceptability and usability;

- improving the ASTRO's behaviour by means of a smart sensor network able to share information with the robot (Ubiquitous Robotics);

- developing an advanced human robot interface based on natural language and able to enhance the usability and interaction of elderly users with the ASTRO robot.

\section{METHODS}

The methodology used in ASTROMOBILE foresaw the involvement of a multidisciplinary team consisted of technology developers, elderly care organizations and designers, which strictly collaborated in order to identify real needs of users and caregivers and to develop the most suitable and appropriate technological solution. The development of the ASTROMOBILE System was scheduled in three phases: (1) the initial end-user requirements' analysis to address criteria of acceptability and usability from the beginning of the design, (2) the physical, hardware and software architectural integration of the entire ASTROMOBILE System and (3) the final experimental evaluation in the Peccioli Living laboratory with elderly people.

\section{A. Analysis of end-users' needs and attitude (Design phase)}

At the beginning of the Experiment, the ASTRO appearance and functionalities were studied and designed with the User Centred Design approach and involving a group of 11 elderly volunteers $65+$ in order to identify a concept responding to usability and acceptability criteria. A well structured program of interviews, focus groups and workshops were organized to point out end-users' needs, considering life styles, habits, needs, quality of life, pathologies, cognitive and/or motor deficits, and the robot's appearance, considering the properties of affordance, safety, aesthetics, friendliness and usefulness. Based on these findings, the ASTRO robot was conceived with a well defined appearance and with appropriate functional capabilities (Table 1).

\section{B. Integration of ASTROMOBILE System (Integration phase)}

The integration of the ASTROMOBILE System covered different aspects, not only technical, but also design and interfaces. By technical point of view, the ASTROMOBILE System was conceived as an interoperable solution based on a ZigBee Wireless Sensor Network, an Inertial Measurement Unit, a mobile robotic platform and appropriately vocal and visual interfaces connected through a Data Communication Bus (see "Instruments" for details). The most demanding interface was the natural language with speech recognition

and vocal synthesis. In order to develop it with the appropriate language (Italian in this case) the eleven elderly recruited during the design phase, were also asked to participate in a developmental session to train the speech recognition software to understand the most common Italian words. The proprieties studied during the design phase brought to a well defined shape of robot, which was therefore modelled with a plastic gray cover, decorated with coloured soft tissues on the body and an anthropomorphic head with eyes illuminated by coloured LEDs (green: standby and ready for a service; blinking blue: working; red: critical situation). According to elderly desires, the cover was also equipped with a handle to support user to stand up or walk and a glove box for small objects.

\section{Evaluation protocol (Evaluation phase)}

During the evaluation phase, 16 elderly people $65+(4$ men and 12 women with 75 main age), including the 11 elderly of the design phase, were involved to use the ASTROMOBILE System in the previous described scenarios, realistically arranged in the Peccioli Living Laboratory, and were asked to complete a questionnaire with multiple-choice and open questions. The experimentation was carried out to test not only the technical performance of the ASTROMOBILE system but also to evaluate the perception of end users about the usability and the acceptability of the system.

The main aspects investigated during the evaluation firstly regarded the functionalities of the system, i.e. the utility, the duration, the usability and the satisfaction. Then also the aesthetics of ASTRO was investigated in order to understand the beautifulness and friendliness of the robot in terms of dimensions, materials, colours and safety perception.

TABLE I. FUnCTIONAL CAPABILITIES OF THE ASTROMOBILE SYSTEM

\begin{tabular}{|l|l|}
\hline \multicolumn{1}{|c|}{$\begin{array}{c}\text { Functional } \\
\text { capabilities }\end{array}$} & \multicolumn{1}{c|}{ Experimental Scenarios } \\
\hline \multirow{2}{*}{$\begin{array}{l}\text { User needs ASTRO } \\
\text { to carry out some } \\
\text { daily activities }\end{array}$} & $\begin{array}{l}\text { User calls ASTRO because he/she needs a physical } \\
\text { help to stand up from the sofa or the bed. }\end{array}$ \\
\cline { 2 - 3 } objects set on the ASTRO's pocket (water, TV \\
remote controller, etc.).
\end{tabular}


Finally, in order to deeply assess the usability of the ASTROMOBILE System, the control interfaces were tested to measure the easiness of using the touch screen, the vocal commands through robot microphone and Smartphone and of understanding the coloured light feedbacks. In addition, the senior volunteers were also asked about general questions related to the general utility of the system, its adaptability in their daily environments, its suitability to their personal habits and life styles and the possibility to purchase it.

\section{INSTRUMENTS}

\section{A. Architecture of the ASTROMOBILE System}

The ASTROMOBILE System was conceptually implemented as a modular architecture structured in four different layers (Fig. 1):

- the physical layer, composed of the hardware components, such as the Mobile Platform, Wireless Sensor Network, Touch Screen, Speaker and Microphone, Smartphone, Inertial Measurement Unit and PC;

- the low-level control process layer, composed of six functional modules, such as Locator, Navigator, Sensor, GUI, Speech and Inertial;

- the high-level coordination layer, composed of the Astrologic module, that was conceived to implement easy algorithms of context awareness and decision making and manage high-level tasks, such as "agenda and reminder", "web interface control" and "Skype-like applications";

- the service layer, represented by the different services that the system was able to provide (Table I).

The six modules of the low-level control process layer were assigned to a specific function and strictly interconnected to each other:

Locator: the Locator module was represented by a ZigBee Localization Network that was able to estimate the positions of robot and end-user in the domestic environment by using both a range based and a range free localization procedure. The ASTRO's and end-user's position was estimated measuring Received Signal Strength Indicator (RSSI) from the messages exchanged between mobile wearable nodes, placed on the robot and the user, and fixed anchor nodes in the environment. Localization was addressed at two levels, i.e. infra-room localization and inroom localization. Firstly a simple range free localization algorithm compared RSSI values from all anchors to identify the occupied room by using a threshold algorithm [10]. Then, a range based localization method was used for a more precise "in-room" user localization. Anchors localization workspace was focused over the most probable user trajectories in the activity of daily life (e.g in front of the bed or near the household appliance) to simplify localization algorithms and a Hata propagation model was used for useranchor relative distance estimation.
Navigator: the Navigator module was implemented to control the autonomous movement of robot and to acquire information from sensors installed on the robot, such as odometry and laser ${ }^{1}$. Navigation was based on two algorithms: Wavefront for the global navigation, which was able to generate a sequence of waypoints that had to be reached by the robot [11], and WFH+ for the local navigation and obstacle avoidance [12].

Sensor: the Sensor module was represented by a ZigBee Sensor Network, maintained by a PC server placed on the environment, which was able to collect information coming from environmental and wearable sensors and provide some communication actions for remote users, robot and end-user.

GUI: the GUI module consisted of a graphical user interface including multi level submenus for easily providing functionalities to end-users, such as multimedia functionalities (news, music and video), Skype calls to relatives or caregivers and monitoring of a desired area of the home. The GUI was also used as interface during dialogues between robot and user (described in the scenarios), as drug reminder or alerts. User could navigate inside different screens (or menus) using a touch screen or by vocal commands processed by the speech module.

Speech: the Speech module represented the natural language interface between the end-user and the robot by means of appropriate tools of speech recognition and vocal synthesis. Speech recognition was based on the Simon opensource speech recognition program, produced by Simon Listens ${ }^{2}$. The speech synthesis was a Text-To-Speech based on MaryTTS System.

Inertial: the Inertial module was a data logger for inertial data (3-axis accelerometers, gyroscopes and magnetometers) coming from the IMU (Inertial Measuring Unit) placed inside the head of the robot.

A client-server communication between modules was developed using a shared communication software bus, using the D-Bus system ${ }^{3}$.

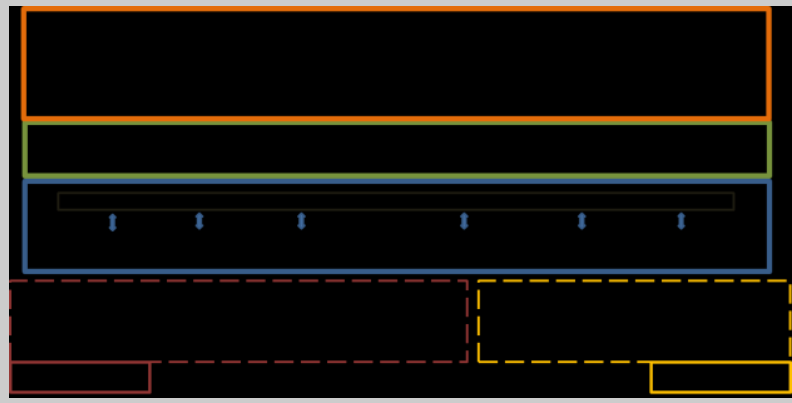

Figure 1. Architecture overwiev of the ASTROMOBILE System

${ }^{1}$ Navigator communicated with the robot actuators and sensors by means of the Player middleware, http://playerstage.sourceforge.net.

2 Simon Listens official web page http://simonlistens.org/index.php? $\mathrm{id}=122 \& \mathrm{~L}=1$.

${ }^{3} \mathrm{D}$-Bus system is a "message bus system, a simple way for applications to talk to one another", D-Bus web page,

http://www.freedesktop.org/wiki/Software/dbus. 


\section{B. Hardware components}

ASTRO robot was based on the Scitos G5 platform (Metralabs GmbH, Germany). The platform was characterized by a very low balance point and an high payload $(50 \mathrm{Kg})$; this features made the robot very stable and difficult to overturn. Perception of surrounding environment was performed by using an additional laser drive (Laser SICK S 300) and an external USB connected ZigBee node allowed the robot to interact and exchange information with the WSNs of the AmI. Moreover, robot was equipped with an additional external IMU (see inertial paragraph).. Concerning safety, SCITOS G5 was equipped with a bumper and a couple of emergency-stop buttons to the stop motor. About Human-Machine-Interface (HMI) a touch screen for directly use of embedded PC was added; besides, as an additional feedback, three set of LEDs were used as an additional feedback about task in progress.

AmI was conceived to expand the sensing capability of the robot improving the situation awareness and to deliver localization based services to the user. It consisted of two ZigBee wireless sensor networks and a PC for data gathering and processing. The two networks, called Localization Network (LN) and Service Networks (SN) provided data for the Locator and Sensor modules, working on different ZigBee channels to avoid medium access conflicts and interferences.

LN worked on ZigBee channel 24 and was composed of fixed anchor nodes and a mobile node. Anchors was equipped with HG2409P directive horizontal linear polarized $\rightarrow$ antenna (HyperGain). The antennas boreside were installed to spot with the main lobe the more frequently accessed areas of the house. The mobile node with an omnidirectional horizontal polarized antenna was worn by the user. Mobile Node and Anchors were continuously exchanging messages each other and were able to estimate the RSSI on such messages, which was related to the distance between them. This localization approach was chosen because of low cost of ZigBee modules, versatility and interoperability with other technologies, low power consumption, low dimensions of sensor modules and so low invasivity in the domestic space and good wearability.

SN worked on channel 26 to monitor the environment by means of appropriate sensors on the doors, beds and sofa. In particular, the information coming from the sensors on the bed and sofa was also used to identify the presence of a subject and combined with data from LN.

\section{RESULTS}

\section{A. The ASTRO first appearance}

The study on the design of ASTROMOBILE System and on how to make it usable and acceptable according to elderly users' attitude and needs allowed to implement domestic services with affordance, safety, aesthetics, friendliness, and usefulness properties. Starting from these proprieties and considering the functionalities identified in this project, the main structural and aesthetic characteristics, such as shape, colour and material, were identified. In addiction some accessories and tools were included in the design in order to make the ASTRO robot more acceptable and usable. The ASTRO's appearance was conceived to immediately show its functionalities (affordance), to be perceived safe (safety), and familiar with an opportune combination of colours and soft / rigid materials (aesthetics), to be emotionally accepted (friendliness) and to be concretely useful in daily activities (usefulness).

\section{B. Experimental Scenarios}

The ASTROMOBILE System was tested according to the services listed in Table I, that were autonomously provided by the robot to the users. In order to describe how the system worked, three examples are considered in detail in this paper. A typical scenario was characterized by the intention of end-user to call ASTRO to request help in some action (Table II). Another typical scenario was characterized by intention of robot to autonomously move to the end-user to help him/her (Table III). Finally a typical scenario is characterized by the intention of caregiver to remotely assist end-user by tele-operating the robot (Table IV).

\section{Scores for acceptability and usability evaluation}

During the experimental sessions, seniors involved in the project had the possibility to concretely test what ASTROMOBILE system was able to do for them (Fig. 2). In order to evaluate its acceptability and usability, subjects were asked to rate the aspects reflecting the main functional capabilities of the ASTROMOBILE System (Fig. 3).

TABLE II. DESCRIPTION Of THE SCENARIO IN WHICH END-USER CALls The RoBot To REQUEST HelP

\begin{tabular}{|c|c|}
\hline Actors & Actions \\
\hline End-User & User sits on the sofa \\
\hline \multirow[t]{3}{*}{$\begin{array}{l}\text { ASTROMOBILE } \\
\text { System }\end{array}$} & $\begin{array}{l}\text { ZigBee presence sensor on sofa transmits information to } \\
\text { the Sensor module }\end{array}$ \\
\hline & Sensor module pushes this information to the Locator \\
\hline & Locator estimates and refreshes user position \\
\hline End-User & $\begin{array}{l}\text { User calls ASTRO robot to stand up from sofa, using the } \\
\text { smartphone }\end{array}$ \\
\hline \multirow[t]{6}{*}{$\begin{array}{l}\text { ASTROMOBILE } \\
\text { System }\end{array}$} & $\begin{array}{l}\text { Vocal commands are sent from the smartphone to the } \\
\text { Speech module }\end{array}$ \\
\hline & $\begin{array}{l}\text { Speech module recognizes the command and sends it to } \\
\text { Astrologic }\end{array}$ \\
\hline & $\begin{array}{l}\text { Astrologic checks robot availability and sends the } \\
\text { command «Go to user» to the Navigator }\end{array}$ \\
\hline & Navigator asks to Locator the user position \\
\hline & $\begin{array}{l}\text { Navigator moves the robot to the given position and } \\
\text { informs Astrologic the position is reached }\end{array}$ \\
\hline & $\begin{array}{l}\text { Navigator gets information from Inertial module to } \\
\text { correct robot current position }\end{array}$ \\
\hline End-User & $\begin{array}{l}\text { User cooperates with the system giving simple movement } \\
\text { voice commands (as «move forward, turn right») to } \\
\text { accomplish the task as the user really need }\end{array}$ \\
\hline \multirow{3}{*}{$\begin{array}{l}\text { ASTROMOBILE } \\
\text { System }\end{array}$} & Speech module recognizes user commands and pass them \\
\hline & directly to the Navigator \\
\hline & $\begin{array}{l}\text { Navigator moves the robot following user vocal } \\
\text { commands }\end{array}$ \\
\hline End-User & The user stands up from sofa using the robot handle \\
\hline
\end{tabular}


TABLE III. DESCRIPTION OF THE SCENARIO IN WHICH AUTONOMOUSLY HELPS USER IN APPROPRIATE SITUATIONS

\begin{tabular}{|l|l|}
\hline \multicolumn{1}{|c|}{ Actors } & \multicolumn{1}{c|}{ Actions } \\
\hline End-User & User is cooking in the kitchen \\
\hline $\begin{array}{l}\text { ASTROMOBILE } \\
\text { System }\end{array}$ & $\begin{array}{l}\text { ZigBee anchors provide RSSI to locate the user in the } \\
\text { kitchen to the Locator module }\end{array}$ \\
\cline { 2 - 2 } & ZigBee sensor on door recognizes the door open \\
\cline { 2 - 2 } & Sensor module informs Astrologic about door status \\
\cline { 2 - 2 } & $\begin{array}{l}\text { Astrologic checks robot availability and sends the } \\
\text { command «Go to user» to the Navigator }\end{array}$ \\
\cline { 2 - 2 } & $\begin{array}{l}\text { Navigator informs Astrologic that the robot approaches } \\
\text { reached position }\end{array}$ \\
\cline { 2 - 2 } & $\begin{array}{l}\text { Astrologic uses both GUI and Speech to alert the user } \\
\text { about the door status }\end{array}$ \\
\hline End-User & $\begin{array}{l}\text { The user confirms to be aware about the critical situation } \\
\text { touching the screen of the robot }\end{array}$ \\
\hline
\end{tabular}

TABLE IV. DESCRIPTION OF THE SCENARIO IN WHICH THE CAREGIVER HELPS USER BY REMOTELY USING THE Rовот

\begin{tabular}{|c|c|}
\hline Actors & Actions \\
\hline End-User & User is lying in bed \\
\hline \multirow[t]{7}{*}{$\begin{array}{l}\text { ASTROMOBILF } \\
\text { System }\end{array}$} & $\begin{array}{l}\text { ZigBee presence sensor provides bed occupancy status to } \\
\text { the Sensor module }\end{array}$ \\
\hline & $\begin{array}{l}\text { Locator uses information from Sensor to estimate user } \\
\text { position }\end{array}$ \\
\hline & $\begin{array}{l}\text { Caregiver uses the web interface to assess the user } \\
\text { (moving to user and activate the webcam) }\end{array}$ \\
\hline & Web interface activates the Astrologic \\
\hline & $\begin{array}{l}\text { Astrologic checks robot availability and sends the } \\
\text { command «Go to user» to the Navigator }\end{array}$ \\
\hline & $\begin{array}{l}\text { Navigator informs Astrologic that the robot approaches } \\
\text { requested position }\end{array}$ \\
\hline & $\begin{array}{l}\text { Astrologic activates the robot webcam and starts a Skype } \\
\text { call to connect the user with the caregiver }\end{array}$ \\
\hline
\end{tabular}

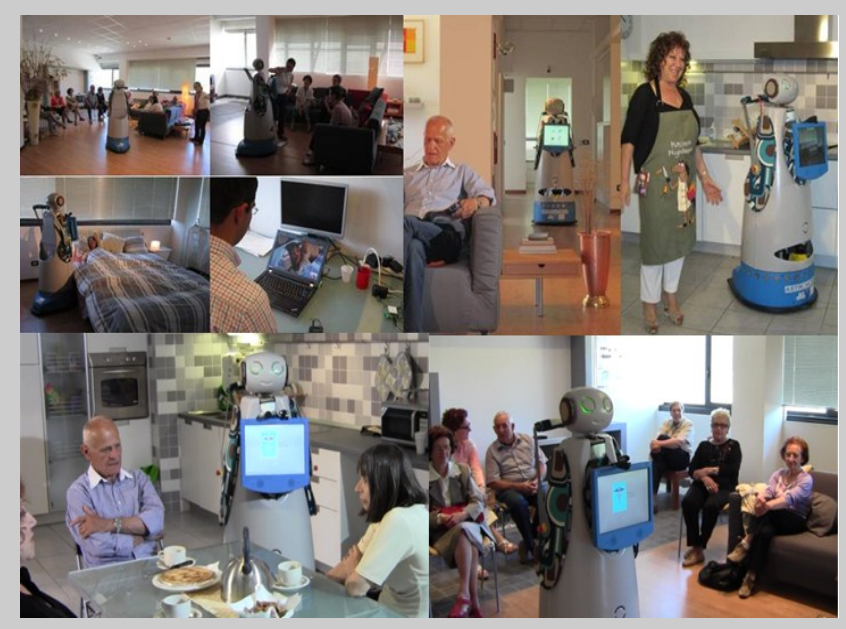

Figure 2. Experimental sessions with elderly people in the Peccioli Lab.

General impression was positive because volunteers appreciated the utility of services which the robotic system provided (i.e. drug reminding, support for standing up, alert in case of opened door, etc.) and also the increasing of safety that they could have from the remote control of caregiver. Most of the functional capabilities were considered very useful with the exception of the entertainment function (Fig. $3 a)$ because they believe that television and radio already satisfied this necessity and the robot could be used in more important tasks. During the experimentation the ASTRO system properly worked and, asking users about the duration of the tasks, they said that the duration of tasks was too long (Fig. 3b).

This perception was mainly due to the velocity of robot navigation that was set low to guarantee the safety of the persons. About the easiness of using the ASTROMOBILE system, interviewees were negative for the scenarios of reminder, communication and entertainment (Fig. 3c).

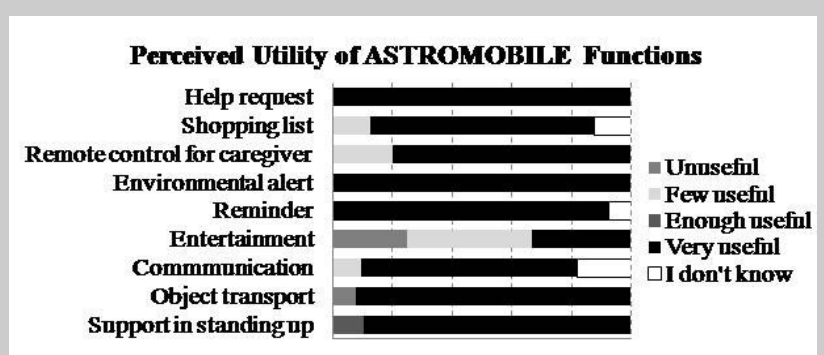

$0 \% 20 \% 40 \% 60 \% 80 \% 100 \%$

(a)

\section{Perceived Duration of ASTROMOBILE scenarios}

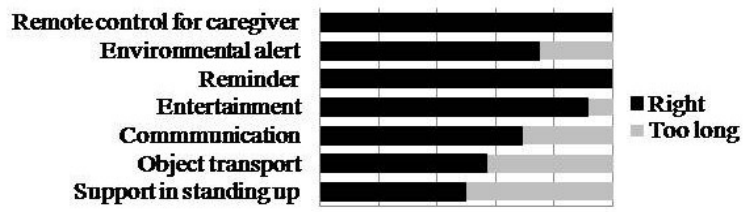

$0 \% 20 \% 40 \% 60 \% 80 \% 100 \%$

(b)

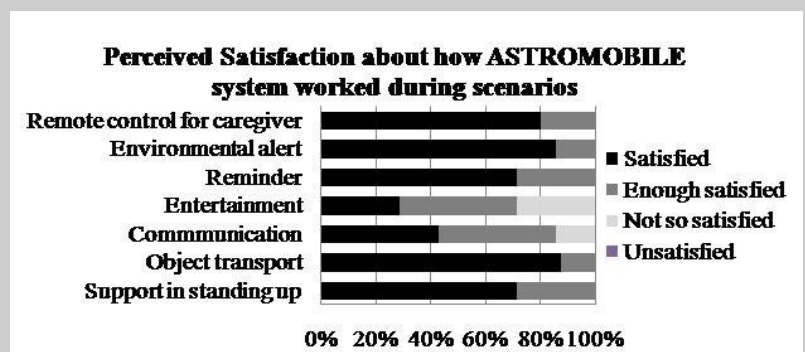

(c)

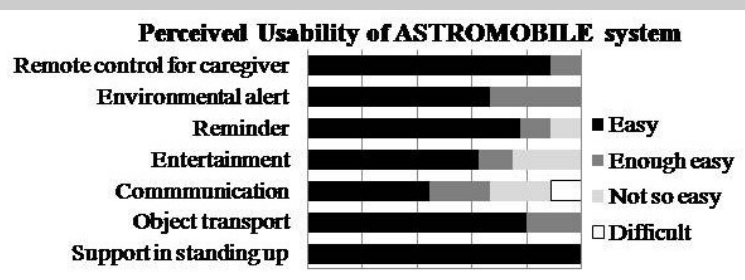

$0 \% 20 \% 40 \% 60 \% 80 \% 100 \%$

(d)

Figure 3. Results of the experimental sessions with elderly people,where the perceived utility, usability, satisfation of the system and the perceived duration of service were investigated.

\section{$54 \mathrm{pt}$} 0.75 in $19.1 \mathrm{~mm}$ 
For communication and entertainment this perception was caused by the fact that the function screen, differently from the main menu screen, had more than four options (list of contacts and list of communication types) and therefore it was perceived not so clear and readable; about the reminder function, the users perceived the navigation in different screens not so easy and furthermore the robot provided the audio messages about the memo with some delay, while elderly subjects expected a more quick and direct vocal communication.

Asking elderly person if they were satisfied about how ASTROMOBILE system worked in the scenarios they replied positively for most of scenarios; the unique "not satisfied" answers were related to entertainment, because the audio of ASTRO speakers were too noisy, and communication scenarios (Fig. 3d). In relation to the ASTRO aesthetics, all the volunteers agreed in feeling ASTRO nice, friendly and safe but the $64 \%$ of them think that ASTRO is still too big for moving in their own apartment. About the ASTROMOBILE interfaces tested by volunteers, they perceived the interfaces based on vocal commands not always easy to use because sometimes the speech recognition module didn't work properly so in that case they should repeat the vocal commands and furthermore the button on the Smartphone screen was too small. However asking subjects about their favourite interfaces, most of them pointed out the vocal interfaces because they were perceived more natural. At the end of the questionnaire there were some general questions about the ASTROMOBILE system; most of elderly subjects thought that ASTRO was useful and also that it could integrate enough in their life style. A very interesting aspect emerged from the question about a possible ASTRO purchase: only two persons (7\%) excluded completely this opportunity.

\section{DISCUSSIONS}

This work demonstrated that the robotic technology for "ageing well" applications is nowadays promising and feasible. The real and concrete involvement of elderly with a user-centred design approach allowed facing developmental aspects that made ASTRO very near to a pre-market solution. In addition the experimentation with elderly in realistic settings allowed to identify also other possible functionalities, such as recognizing persons who come in the house, understanding what the persons do (i.e. if the user takes drugs), working as alarm clock and manipulating objects and reaching high points.

Elderly were trained to use ASTRO and this gave the possibility to better understand how to implement facilitated interfaces and to spread the culture of considering the companion robot similar to a household appliance.

ASTRO was developed with an interoperable integration of mature technological and standardized solutions already available on the market, such as the mobile robotic platform (Metralabs, Germany), Wireless Sensor Network (ST Microelectronics, Italy), Software Interface (Simon Listens, Austria), robot and environment OS (Win7 and Ubuntu) and other minor components.
The cooperation between ASTRO and the AmI allowed to improve the quality of services provided to end-users. For example the ZigBee Localization Network was used to improve the indoor localization capabilities by measuring the RSSI signal between the mobile nodes, i.e. the robot and the end-user, and the anchor nodes in the environment and estimating their respective positions; this allowed a sort of awareness, thanks to which the robot was aware of the position of the end-user to reach in the workspace, improving the quality of services. Thanks to the shared information between the ASTRO Robot and the Wireless Sensor Networks and the effectiveness of the ASTROMOBILE system, that was able to exploit sensing, acting and interacting abilities with respectively the wireless sensor networks, the robot and the advanced user-robot interfaces, the capabilities of the entire system to provide high quality services to elderly users were particularly improved.

Focused on acceptability and usability, the paper did not treat an appropriate analysis of the dependability of system, that will be evaluated in the next work.

\section{REFERENCES}

[1] European Robotics Technology Platform (EUROP), Robotic Visions To 2020 And Beyond - The Strategic Research Agenda For Robotics In Europe, July 2009.

[2] T. Plowman, D. Prendergast, S. Roberts, and J. Sherry, The Global Aging Experience Project. Ethnographic Research, Intel research, 2010.

[3] European Commission - Economic Policy Committee, The 2012 Ageing Report: Economic and budgetary projections for the $27 \mathrm{EU}$ Member States (2010-2060), 2012.

[4] G. van der Broek, F. Cavallo, and C. Wehrmann, AALIANCE Ambient Assisted Living Roadmap, vol. 6, IOS Press, 2010.

[5] The MOBISERV Project (An Integrated Intelligent Home Environment for the Provision of Health, Nutrition and Mobility Services to the Elderly) - http://www.mobiserv.eu/index.php?lang=en

[6] The KSERA Project (Knowledgeable SErvice Robots for Aging) http://ksera.ieis.tue.nl/

[7] The SRS Project(Shadow Robotic System for Independent Living) http://srs-project.eu/

[8] The Florence Project (Multi Purpose Mobile Robot for Ambient Assisted Living) - http://www.florence-project.eu

[9] The CompanionAble Project (Integrated Cognitive Assistive \&Domotic Companion Robotic Systems for Ability \& Security) http://www.companionable.net/

[10] Care-O-Bot 3 - http://www.care-o-bot.de/english/index.php

[11] Robot Maid - http://www.lunegate.com

[12] F. Cavallo, M. Aquilano, M. Bonaccorsi, I. Mannari, M.C. Carrozza, and P. Dario, "Multidisciplinary approach for developing a new robotic system for domiciliary assistance to elderly people", Proc. $33^{\text {rd }}$ Annual International Conference of the IEEE Engineering in Medicine and Biology Society, EMBC 2011, Boston, US, September 2011.

[13] S. M. Lavalle, Planning algorithms, Cambridge University Press, Chambridge, UK, 2006.

[14] I. Ulrich, and J. Borenstein, "WFH+: reliable obstacle avoidance for fast mobile robots", Proc. 1998 IEEE International Conference on Robotics and Automation, ICRA 98, Leuven, Belgium, pp.15721577,May, 1998. 